\title{
SEARCH FOR EXTRA DIMENSIONS WITH ATLAS AT LHC
}

\author{
K. BENSLAMA \\ Nevis Laboratories, Columbia University \\ 136 S. Broadway, \\ Irvington, $N Y$ 10533, USA \\ E-mail: benslama@nevis.columbia.edu
}

\begin{abstract}
Theories with extra space dimensions aiming at resolving the hierarchy problem have recently been developed. These scenarios have provided exciting new grounds for experimental probes. A review of some recent studies done by the ATLAS collaboration on the sensitivity of the detector to various extra dimension models is reported in this document.
\end{abstract}

\section{Introduction}

The complete unification of particle interactions might require the presence of additional spacial dimensions, three of which are within the reach of our senses and the others being compactified at distances of the order of $10^{-32}$ m. In a model inspired by string theory, Arkani-Hamed, Dimopoulos and Davali (ADD) suggested that there is only one fundamental scale in nature; the electroweak scale ${ }^{1}$. In this setup gravity appears much weaker than other forces because it can propagate in extra spacial dimensions and hence its flux lines are diluted, while the standard model fields are confined to the three space dimensions. Since then, the idea of extra dimensions has been a subject of intense phenomenological studies.

\section{ATLAS Studies}

The sensitivity of the ATLAS detector to a number of phenomenological models has been studied. The results of some of these studies are reported in the following sections. Most of these studies have used a parameter-based fast Monte Carlo, ATLFAST, to simulate the effects related to the detector. ATLAS is expected to accumulate $100 \mathrm{fb}^{-1}$ data in a high luminosity run of the LHC per year. 


\subsection{Large Extra Dimension}

In the presence of large extra dimensions, gravitons will appear as towers of massive Kaluza-Klein (KK) states from the 4-D point of view. Since the coupling of gravitons to matter is very weak, direct production of KK gravitons will results in signals with large missing transverse energy in the detector. Final states of jets plus missing energy and photons plus missing energy ${ }^{2}$ have been simulated in the ATLAS detector. Figure 1 shows signal and backgrounds distributions. After one year of running at the design luminosity, the LHC can probe the mass scale of the theory up to 9, 7 and $6 \mathrm{TeV}$ if there are respectively two, three or four extra dimensions. Running the LHC at different center-of-mass energies could help to find out the number of extra dimensions.

A virtual production of the KK gravitons will result in the modification of the Drell-Yan cross section. Studies have been done with photon and lepton pairs in the final state. A one year run at high luminosity should have sensitivity up to $8(7) \mathrm{TeV}$ for a number of extra dimensions equal to $5(2)^{3}$.

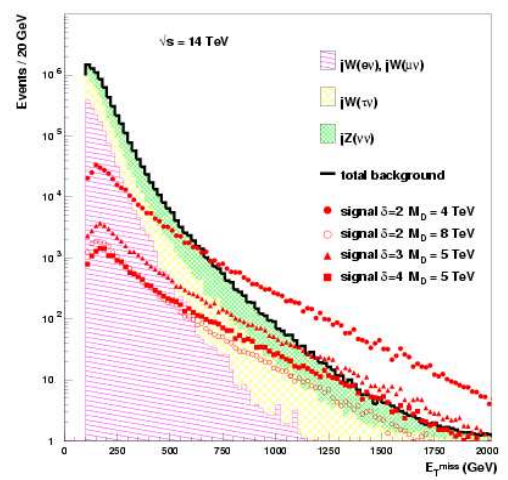

Figure 1. Distribution of missing transverse energy for signal and background events

\section{2. $\mathrm{TeV}^{-1}$ Extra Dimension}

An interesting variation to the above scenario is the one in which only the standard model fermions are confined to the three space dimensions, while there can be Kaluza-Klein excitations of the gauge boson in the presence of small extra dimension. In ATLAS, we have performed a detailed study 
of the leptonic signatures for the production of Kaluza-Klein excitations of the $\gamma$ and $\mathrm{Z}$ in models with $\mathrm{TeV}$-scale extra dimensions ${ }^{4}$. The production and decay of the excitations were fully simulated, and the resulting particles were passed through a parametrized simulation of the ATLAS detector. Figure 2 shows the invariant mass distribution of $e^{+} e^{-}$pairs for the standard model (full line) and for models M1 (dashed line) and M2 (dotted line) for which fermions lie at different fixed points on the wall. With an integrated luminosity of $100 \mathrm{fb}^{-1}$ ATLAS will be able to detect a peak in the lepton-lepton invariant mass if the compactification scale $\left(M_{c}\right)$ is below 5.8 TeV. In the absence of a peak, a detailed study of the shape of the lepton-lepton invariant mass will allow us to observe the interference effect up to $M_{c} \sim 13.5 \mathrm{TeV}$.

Once a peak is observed, an important task is the assessment of the model which has produced the signal. With an integrated luminosity of $300 \mathrm{fb}^{1-}$ and for resonances of mass up to $5 \mathrm{TeV}$, the KK excitations can be distinguished from mass peaks produced by SM-like $Z^{\prime}$ or graviton resonances from the study of the forward backward asymmetry of the leptons in the peak region.

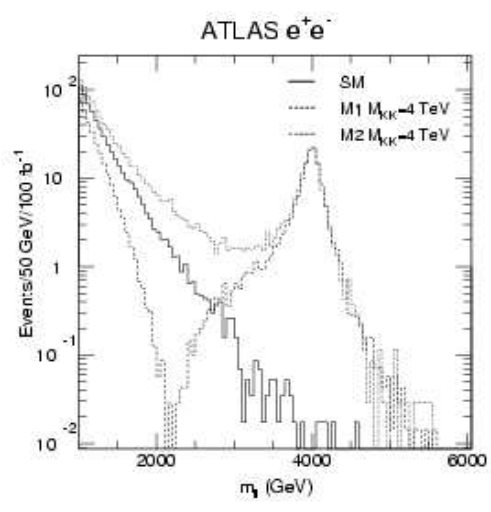

Figure 2. Invariant mass distribution of $e^{+} e^{-}$for the standard model and for models M1 and M2. The mass of the lowest lying KK excitation is $4000 \mathrm{GeV}$. The histograms are normalized to $100 \mathrm{fb}^{-1}$

\subsection{Randall-Sundrum Models}

The Randall-Sundrum model postulates a universe with two 4-D surfaces ( $\mathrm{TeV}$ and Plank brane) bounded by 5-D space-time. The SM fields are 
assumed to be located on one of the two branes (the $\mathrm{TeV}$ brane), while gravity lies in the bulk. The fifth dimension is not large, but the metric is non factorisable, allowing for a resolution of the hierarchy problem, given an appropriate warp factor. The theory admits two types of four-dimensional massless excitations: the usual graviton and a graviscalar, the modulus or radion $(\phi)$. In order to stabilize the size of extra dimensions without fine tuning of parameters, Goldberger and Wise have proposed a mechanism by which the radion acquires a mass, expected to be smaller than the $\mathrm{J}=2$ Kaluza-Klein excitations. The presence of the radion is one of the important phenomenological consequences of these theories of warped extra dimensions.

ATLAS has performed a model independent study of narrow graviton resonance signals ${ }^{5}$. Figure 3 shows the number of $G \rightarrow e e$ events per 4 $\mathrm{GeV}$ mass bin from a graviton resonance along with the expected standard model background. In the context of the RS model, we obtained a 5 sigma sensitivity up to $2.1 \mathrm{TeV}$ of graviton mass in the di-electron production channel. Studies of the angular distribution of the polar angle of electrons show that a spin- 2 resonance can be distinguished from a spin-1 resonance up to a mass of $1.7 \mathrm{TeV}$ with $90 \% \mathrm{CL}$.

ATLAS has also investigated the possibility of observing the radion via its decay into pair of Higgs scalars ${ }^{6}$. Two cases were examined: $\phi \rightarrow h h \rightarrow$ $\gamma \gamma b \bar{b}$ and $\phi \rightarrow h h \rightarrow \tau \tau b \bar{b}$, for radion masses of 300 and $600 \mathrm{GeV}$, for $m_{h}=$ $125 \mathrm{GeV}$ and for an integrated luminosity of $30 \mathrm{fb}^{-1}$. Figure 4 shows the reconstructed mass of the radion for $30 \mathrm{fb}^{-1}$ and for $\Lambda_{\phi}=1 \mathrm{TeV}, \xi=0$, where $\Lambda_{\phi}$ is the vev of the radion and $\xi$ is a parameter describing mixing with the Higgs boson. Limits on the maximal reach in $\Lambda_{\phi}$ were obtained for these two channels. For the $\gamma \gamma b \bar{b}$ channel, the background is negligible and the reach in $\Lambda_{\phi}$ is $2.2(0.6) \mathrm{TeV}$ for $m_{\phi}=300(600) \mathrm{GeV}$, when $\xi=0$. For the $\tau \tau b \bar{b}$ channel, the reach for $\Lambda_{\phi}$ is about $1 \mathrm{TeV}$ for both $m_{\phi}=300 \mathrm{GeV}$ and $m_{\phi}=600 \mathrm{GeV}$, when $\xi=0$, although in the first case, uncertainties in the background shape may result in large systematic errors.

\section{Summary}

The ATLAS collaboration has studied different phenomenological models with extra space dimensions. The experiment will be very sensitive to the presence of these extra dimension and will be able to probe mass scales of 5 to $10 \mathrm{TeV}$, depending on the model. 


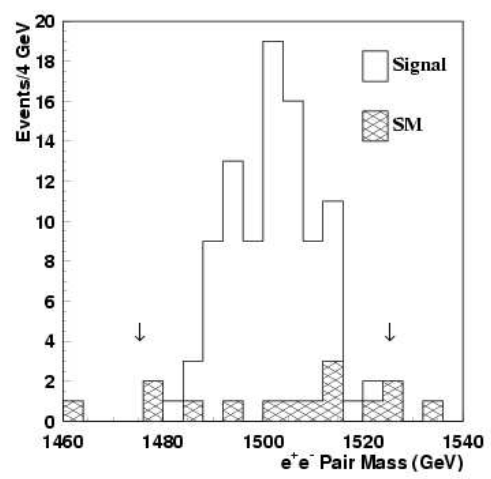

Figure 3. The number of events per $4 \mathrm{GeV}$ mass bin from a graviton resonance, with $m_{G}=1.5 \mathrm{GeV}$, superimposed on the expected standard model background, for $100 \mathrm{fb}^{-1}$ of integrated luminosity.
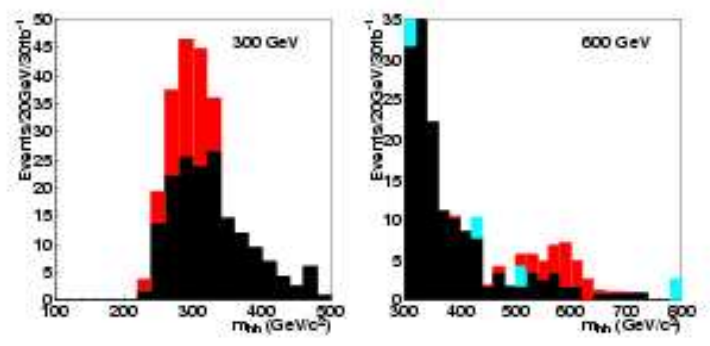

Figure 4. Reconstructed mass of the radion, for $30 \mathrm{fb}^{-1}$ and $\Lambda_{\phi}=1 \mathrm{TeV}, \xi=0$. Left plot: expected signal (light) and $t \bar{t}$ background (dark). Right plot: $t \bar{t}$ background (dark), expected signal (light) and $\mathrm{Z}+$ jets backgrounds (very light).

\section{Acknowledgments}

We gratefully acknowledge enlightening discussions with Georges Azuelos.

\section{References}

1. N. Arkani-Hamed, S. Dimopoulos and G. Dvali, Phys. Lett. B429, 263 (1998)

2. L. Vacavant and I. Hinchliffe, J. Phys. G: Nucl. Part. Phys. 27, 1839 (2001)

3. V. Kabachenko, A. Migakov, A. Zenin, ATL-PHYS-2001-012

4. G. Azuelos, G. Polesello, hep-ph-0204031

5. B. C. Allanach, K. Odagiri, A. Parker and B. Webber, JHEP9(2000) 19

6. G. Azuelos, D. Cavalli, H. Prysiezniak, L. Vacavant, hep-ph/0204031 